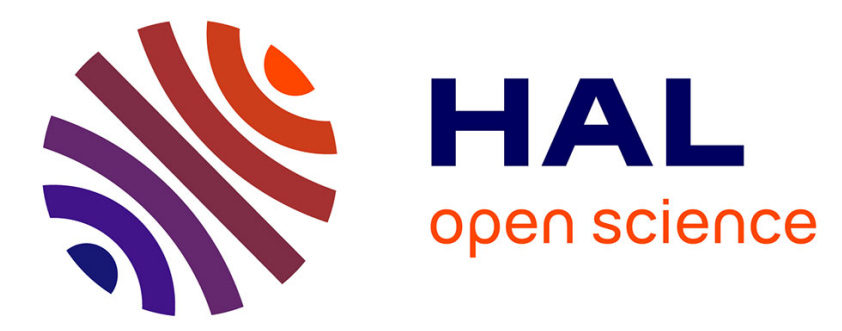

\title{
Acteurs et territoires du développement rural en Allemagne orientale. L'exemple de projets de développement régional dans la Thuringe du Sud-Ouest Christophe Queva
}

\section{- To cite this version:}

Christophe Queva. Acteurs et territoires du développement rural en Allemagne orientale. L'exemple de projets de développement régional dans la Thuringe du Sud-Ouest. Revue d'Etudes Comparatives Est-Ouest, 2007, p. 117-134. halshs-01708876

\section{HAL Id: halshs-01708876 \\ https://shs.hal.science/halshs-01708876}

Submitted on 14 Feb 2018

HAL is a multi-disciplinary open access archive for the deposit and dissemination of scientific research documents, whether they are published or not. The documents may come from teaching and research institutions in France or abroad, or from public or private research centers.
L'archive ouverte pluridisciplinaire HAL, est destinée au dépôt et à la diffusion de documents scientifiques de niveau recherche, publiés ou non, émanant des établissements d'enseignement et de recherche français ou étrangers, des laboratoires publics ou privés. 
Acteurs et territoires du développement rural en Allemagne orientale. L'exemple de projets de développement régional dans la Thuringe du Sud-Ouest

Christophe Queva

\section{Citer ce document / Cite this document :}

Queva Christophe. Acteurs et territoires du développement rural en Allemagne orientale. L'exemple de projets de développement régional dans la Thuringe du Sud-Ouest. In: Revue d'études comparatives Est-Ouest, vol. 38, 2007, ${ }^{\circ} 3$. Le développement rural en Allemagne réunifiée : modèles, contextes, enjeux. pp. 117-134;

http://www.persee.fr/doc/receo_0338-0599_2007_num_38_3_1849

Document généré le 08/06/2016 


\begin{abstract}
Actors and areas in rural development- Regional development projects in southwestern Thuringia Local actors in eastern Germany, who used to abide by the German Democratic Republic's centralized planning, now have to take an active part in development projects under the 1998 Federal Regional Planning Act, which explicitly names "projects of regional development" as new tools for local development. Whether subsidized with public or private funds, these projects, drawn up locally, rely on traditional planning authorities. In particular, the region has become the level for coordinating local projects. The switch from the old to the new system of planning has raised several issues. Seeing actors in terms of relations and networks helps us better understand the many complex projects under way, in particular under regional planning in southwestern Thuringia.
\end{abstract}

\title{
Résumé
}

À l'est de l'Allemagne, les acteurs locaux, marqués par la tradition d'aménagement centralisé héritée de l'ex-RDA, doivent désormais s'investir dans des projets de développement comme la Loi sur l'aménagement du territoire le prescrit depuis sa réforme en 1998. Celle-ci désigne explicitement les " projets de développement régional " comme les nouveaux instruments du développement local. Élaborés pas les acteurs locaux, qu'ils soient publics ou privés, ces projets doivent s'appuyer sur les échelons traditionnels de l'aménagement, en particulier sur la région de planification devenue l'échelon de référence pour coordonner les projets locaux. Toutefois, diverses tensions se manifestent entre ces anciennes et ces nouvelles pratiques de l'aménagement et du développement. Une approche relationnelle et réticulaire de la notion d'acteur permet de clarifier la multiplicité et la complexité des projets à l'œuvre, notamment dans le cadre d'une région de planification : la Thuringe du Sud-Ouest. 


\section{ACTEURS ET TERRITOIRES dU DÉVELOPPEMENT RURal en Allemagne orientale}

\section{L'EXEMPLE DE PROJETS DE DÉVELOPPEMENT RÉGIONAL dans la Thuringe du SUd-OUest}

\section{CHRISTOPHE QUÉVA}

ATER à l'Université M. de Montaigne (Bordeaux III), UMR 5185 du CNRS "Aménagement, développement, environnement, santé et sociétés »-ADES (chrisqueva@hotmail.com)

Résumé : À l'est de l'Allemagne, les acteurs locaux, marqués par la tradition d'aménagement centralisé héritée de l'ex-RDA, doivent désormais s'investir dans des projets de développement comme la Loi sur l'aménagement du territoire le prescrit depuis sa réforme en 1998. Celle-ci désigne explicitement les « projets de développement régional » comme les nouveaux instruments du développement local. Élaborés pas les acteurs locaux, qu'ils soient publics ou privés, ces projets doivent s'appuyer sur les échelons traditionnels de l'aménagement, en particulier sur la région de planification devenue l'échelon de référence pour coordonner les projets locaux. Toutefois, diverses tensions se manifestent entre ces anciennes et ces nouvelles pratiques de l'aménagement et du développement. Une approche relationnelle et réticulaire de la notion d'acteur permet de clarifier la multiplicité et la complexité des projets à l'œuvre, notamment dans le cadre d'une région de planification : la Thuringe du Sud-Ouest. 
Depuis la réforme de la Loi sur l'aménagement du territoire (Raumordnungsgesetz) en 1998, la Region est devenue, en Allemagne, un outil majeur de développement local (regionale Entwicklung) ${ }^{1}$, parallèlement aux approches traditionnelles d'aménagement «par le haut » (Raumordnung) et de planification (Raumplanung). L'idée est de dépasser les territoires traditionnels et emboîtés de l'aménagement - communes, Land$k_{r e i s e^{2}}$, régions de planification ${ }^{3}$, Länder et État fédéral - pour valoriser des espaces plus flous et informels, basés sur la coopération entre acteurs locaux : les Regionen ou régions de projet. Dans ce cadre dit "régional », les acteurs locaux sont appelés à concevoir des projets de dévcloppcment que les instances traditionnelles de l'aménagement, notamment les régions de planification, sont censées coordonner. Les projets ont généralement une dynamique complexe, soucieuse de concilier à la fois les approches patrimoniales et territoriales - par la mise en évidence d'une unité régionale - et les approches innovantes et réticulaires - par la constitution de coopérations informelles, ouvertes, non limitées spatialement. L'idée d'une tension entre territoires et réseaux est d'autant plus prégnante en milieu rural que les notions d'appartenance et de territoire local y sont vivaces et susceptibles d'accentuer ou, au contraire, de s'opposer aux approches en termes de réseaux. De surcroît, à l'est de l'Allemagne, les acteurs locaux ont dû faire face aux mutations particulièrement rapides et profondes de la réunification. Il s'agissait pour eux de passer d'une tradition d'aménagement marquée par les approches territoriales et hiérarchisées (Territorialplanung ${ }^{4}$ ) à cette nouvelle pratique du développement local par le projet, fondée sur la mise en place de réseaux d'acteurs régionaux.

La région de planification de Thuringe du Sud-Ouest ${ }^{5}$, que nous avons choisie comme cadre d'analyse de projets de développement régional, n'est

1. Le paragraphe 13 de la Loi sur l'aménagement (Raumordnungsgesetz) de 1998, intitulé «Verwirklichung der Raumordnungspläne ", désigne explicitement les projets de développement régional (regionale Entwicklungskonzepte) comme les nouveaux outils de mobilisation et de coopération locales.

2. Les Kreise forment, avec les communes, les collectivités de l'échelle locale. On distingue les Stadtkreise, qui correspondent à une agglomération urbaine (Erfurt ou Potsdam par exemple), des Landkreise dont la superficie est plus étendue et la structure de peuplement plus discontinue.

3. Chaque Land est divisé en « régions de planification " (Planungsregionen), chargées traditionnellement de relayer les plans d'aménagement du Land. Ces régions sont appelées « régions d'aménagement » (Raumordnungsregionen) à l'échelle de l'État fédéral. On en compte 97 en Allemagne (données du Bundesamt für Bauwesen und Raumordnung - BBR [Office fédéral de l'aménagement du territoire], 1996).

4. La planification territoriale fait référence aux pratiques centralisées du temps de la RDA ; cf. BÖNisCH et al., 1980.

5.24 entretiens semi-directifs ont été menés en Thuringe méridionale, entre juin et septembre 2005. Ils ont été effectués en allemand auprès des principaux acteurs du développement régional. Notre thèse portant sur les expériences de développement local en France et en Allemagne, ils ont complété d'autres entretiens en Aquitaine (Pays du Libournais/Pays du Périgord noir). Au total, 76 entretiens ont été réalisés. 
pas exempte d'ambiguïtés, d'où un certain nombre de tensions entre anciennes et nouvelles pratiques de l'aménagement et du développement. Elle constitue un espace à dominante rurale, associé à une entité paysagère, identitaire et touristique : la Forêt de Thuringe. Par ailleurs, la région correspond quasiment à l'ancien Bezirk ${ }^{6}$ de Suhl. Du temps de la RDA, celui-ci se trouvait à la périphérie du territoire national, à la frontière avec l'ex-Allemagne de l'Ouest, alors qu'aujourd'hui, la région de planification de Thuringe du Sud-Ouest est géographiquement au centre de l'Allemagne réunifiée. De nouveaux axes majeurs de communication l'intègrent de manière croissantc au reste de la Thuringe et du territoire allemand, notamment en direction de la Bavière et de la Hesse, les deux Länder voisins.

Il convient de s'interroger sur les modalités de la réalisation de projets souples de développement régional dans un contexte caractérisé par de fortes territorialités ${ }^{7}$. Nous verrons, d'abord, dans quelle mesure des acteurs régionaux (locaux) ont su entreprendre des projets et des actions de développement en accord ou en contradiction avec les nouvelles attentes des politiques d'aménagement. Nous nous intéresserons ensuite aux territoires du développement où interviennent ces acteurs : sont-ils fonctionnels, souples et ouverts ou ne font-ils, au contraire, que refléter les territoires administratifs ? Après avoir exposé les ambiguïtés et les tensions locales associées aux mutations des cadres de l'aménagement en Allemagne, nous analyserons, à travers cette étude de cas, les processus de régionalisation en cours, tant au niveau des acteurs que des territoires.

\section{DEuX Visions COMPLÉMENTAIRES OU ANTITHÉTIQUES DE L'AMÉNAGEMENT ET DU DÉVELOPPEMENT?}

Le système d'aménagement traditionnel était défini, aussi bien en RFA qu'en RDA, par la planification, qu'elle fût centralisée à l'échelle du Land pour la première (Landesplanung) ou à celle du territoire national pour la seconde (Territorialplanung). Cette approche verticale de l'aménagement persiste dans l'Allemagne réunifiée. Elle implique la coordination d'échelons territoriaux emboîtés qui planifient chacun à leur niveau : État fédéral, Land, région de planification (Planungsregion), Kreis et commune.

6. La RDA était divisée en 15 Bezirke, administrés de manière centralisée. Chaque Bezirk était organisé autour d'une ville (exemple : Suhl), marquée dès lors par le poids des fonctions administratives.

7. Le terme de «territorialités» est pris ici dans son sens le plus courant en géographie, c'est-à-dire le « rapport individuel ou collectif à un territoire considéré comme approprié » (R. Brunet, R. Ferras et H. Théry, dir., Les mots de la géographie, dictionnaire critique, Paris : Reclus-La Documentation française, 1993, $3^{e}$ édition, p. 481). Les territorialités évoquées sont celles qui concernent l'héritage des territoires de référence (notamment l'ancien Bezirk) ou encore les deux espaces de collines et de moyennes montagnes forestières liées à un sentiment d'appartenance : la Rhön et la Forêt de Thuringe. 
Cependant, dans un contexte général de valorisation du local et des initiatives endogènes ${ }^{8}$, une réforme de la Loi sur l'aménagement du territoire (Raumordnungsgesetz-ROG) a vu le jour en 1998. Elle s'articule autour de deux idées: le passage de la planification au développement spatial (Raumentwicklung) et le renforcement d'un échelon dit « régional ${ }^{9}$ pour mener à bien ce développement. Celui-ci n'est pas associé à un territoire administratif. La loi appelle plutôt les acteurs locaux à sortir des cadres traditionnels de l'aménagement pour proposer des espaces de coopération pertinents fondés sur des contrats et des projets, au détriment des plans et programmes traditionnels ${ }^{10}$; elle préconise ainsi les projets dits de développement régional (regionale Entwicklungskonzepte). Dans ce cadre, la $R e$ gion, au sens de région de projet, est un échelon local lié à des coopérations intercommunales souples, aussi bien au sein d'un Kreis qu'entre plusieurs Kreise, voire entre régions de planification et même entre Länder.

\subsection{LES TENSIONS ENTRE ANCIENNES \\ ET NOUVELLES PRATIQUES DE L'AMÉNAGEMENT}

Le développement dit « régional » apparaît comme une nouvelle conception de l'aménagement «par le bas », accordant une grande place aux acteurs locaux dans l'élaboration des projets de développement. Il illustre un changement récent d'idéologie et de mode d'action que l'on retrouve dans d'autres pays européens, que ce soit en France (Pays, pôles de compétitivité, pôles d'excellence rurale), en Italie (systèmes productifs locaux) ou encore en Hongrie (micro-régions). Ces approches favorisent les «projets», les «innovations " en matière de «développement", l'instauration de nouvelles « gouvernances locales » associées, en Allemagne, à des «managers régionaux ", autant de termes issus du monde de l'économie (Greffe, 2002). Parallèlement aux approches traditionnelles de l'aménagement planifié et encadré, la logique qui s'instaure privilégie une vision concurrentielle. Dans le cas des nouveaux Länder, ces transformations contrastent fortement avec les pratiques de la planification territoriale héritées de l'ex-RDA. À l'apprentissage de la démocratie, accéléré par le transfert et l'imposition du modèle occidental de démocratie locale (Wollmann, 1996; Lozac'h, 2001), s'ajoute, pour les acteurs locaux d'Allemagne orientale, la nécessité de suivre des logiques teintées d'un libéralisme dont ils maîtrisent mal les outils et les règles. À cet égard, les projets pilotes d'aménagement de l'espace (Modellvorhaben der Raumordnung) sont assez révélateurs : après un

8. Nous pensons notamment au poids des politiques régionales européennes dans le renforcement des initiatives locales (par exemple, le programme LEADER dans les espaces ruraux) et à des processus similaires dans d'autres pays européens : politique des Pays en France, comarcas en Espagne, micro-régions en Hongrie, etc.

9. Pour une clarification de la notion de Region en Allemagne et de ses différents usages en géographie et dans les pratiques de l'aménagement, voir QuÉvA, 2007a.

10. Pour plus de détails, voir le $§ 13$, Verwirklichung der Raumordnungsplän, de cette loi. 
appel à projets, l'État fédéral sélectionne ceux qui lui paraissent les plus novateurs. Leur réalisation bénéficie de subventions et, en cas de succès, ils peuvent éventuellement devenir des modèles d'aménagement transposables dans d'autres espaces. Cet exemple illustre bien l'interpénétration des deux logiques d'aménagement et de développement, ce qui n'est pas sans susciter une tension entre structures formelles et informelles et, plus généralement entre territoire et réseau. On pourrait analyser cette idée à trois niveaux de lecture.

Sur le plan spatial, en premier lieu, la Region, comme échelle du développement, fait appel à deux types d'espaces associés aux deux pratiques du développement et de l'aménagement (Quéva, 2007a). Le premier est celui des « projets de développement régional » ou régions de projet, espaces coopératifs, souples et informels de l'ordre du réseau. Ces projets sont ensuite coordonnés par un autre échelon qui se trouve dès lors renforcé : celui de la « région de planification ». Celle-ci continue en effet d'exister à la fois en tant qu'échelon-relais du Land et échelon de coordination des projets locaux.

En second lieu, en ce qui concerne les acteurs régionaux chargés de la réalisation des projets de développement régional, la même tension est perceptible entre les nouveaux acteurs du développement et les acteurs traditionnels et territorialisés de l'aménagement. Les premiers correspondent aux sphères du développement au sens large - associations, entreprises, techniciens des territoires (managers régionaux), etc. - et s'inscrivent dans une organisation plutôt horizontale et réticulaire où la notion de hiérarchie est abandonnée au profit de celles d'interdépendance et d'interrelation. La hiérarchie des acteurs n'est pas éliminée pour autant, les acteurs traditionnels de l'aménagement - les maires, les Landräte (élus des conseils exécutifs des Landkreise) et les représentants du Land pour les questions d'aménagement (région de planification) - constituant toujours le cadre de référence de l'action locale.

Enfin, dans leur contenu, les projets de développement régional en tant que tels oscillent entre un idéal de fonctionnalité spatiale, en particulier dans le cadre de réseaux de villes organisant l'espace régional, et une certaine patrimonialisation liée au renforcement de régions identitaires comme nous aurons l'occasion de le préciser par la suite.

On peut donc retenir, en termes de cadrage, que deux visions de l'aménagement et du développement coexistent aujourd'hui en Allemagne : une vision traditionnelle, territorialisée et hiérarchisée, et une vision novatrice, plus souple et informelle, tournée vers le réseau. Entre les deux, la dynamique des relations crée des tensions pouvant déboucher sur de réelles expériences de coopération mais aussi sur des conflits susceptibles de freiner le développement local. 


\subsection{La multiplication des Regionen en Thuringe du Sud-Ouest : COOPÉRATIONS ET CONFLITS}

La Thuringe, située au nord de la Bavière et à l'est de la Hesse, est l'un des six nouveaux Länder. Dans l'imaginaire collectif, elle est associée à la Forêt de Thuringe, espace de moyenne montagne dont la ligne de crête est parcourue par un célèbre chemin de randonnée : le Rennsteig. Celui-ci sépare la Franconie, au sud, du reste de la Thuringe, au nord. Comme le montre le Document 1, les principales villes de Thuringe s'organisent le long d'un axe est-ouest allant de Gera à Eisenach en passant par Jena, Weimar et Erfurt. Concernant la région de planification étudiée, on peut retenir qu'elle présente une unité réelle autour de la Forêt de Thuringe, dont elle forme le versant sud. D'autres éléments paysagers la caractérisent : la vallée de la Werra et la Rhön, espace de collines correspondant à une réserve de biosphère. La Thuringe du Sud-Ouest est organisée autour de deux villes moyennes de 40000 habitants : Suhl, capitale de l'ancien Bezirk, et Eisenach, célèbre pour la Wartburg, haut-lieu par excellence de l'identité allemande" ${ }^{\prime}$. Du point de vue du découpage, elle est l'une des quatre régions de planification du Land de Thuringe chargées de mettre en pratique les plans régionaux conformément aux directives du Land. Chacune regroupe plusieurs Kreise. La Thuringe du Sud-Ouest compte ainsi quatre Landkreise et deux villes-Kreis (Suhl et Eisenach). Ce sont là les territoires «traditionnels » de l'aménagement, conformément à la pratique de la planification.

Avec la réforme de la Loi sur l'aménagement du territoire en 1998, de nouvelles formes d'aménagement sont apparues, prônant le développement par le bas. Elles entendent favoriser le «développement régional » qu'elles associent à l'objectif du développement durable ${ }^{12}$. En Thuringe, comme ailleurs en Allemagne, des projets de développement régional sont mis en place en tant que « nouveaux instruments, informels, souples et d'initiative locale » $(B B R)^{13}$. Ils reposent sur la coopération de partenaires privés (associations, entreprises, organismes touristiques, etc.) et publics (communes, Kreise) en vue d'actions concrètes d'ordre thématique telles que la mise en réseau d'acteurs touristiques, la construction d'une zone d'activité, la création d'un réseau régional de transports en commun. Dans le cadre étudié, les projets régionaux sont souvent indépendants des frontières administratives comme l'indique le Document 2. Ils sont intercommunaux et dépassent les limites des Kreise à l'image de l'Association de villes de Thuringe du Sud (Städte-verbund Südthüringen) qui s'étend sur deux Land-

11. La château fort de la Wartburg renvoie aux concours des Minnesänger, évoqués par Richard Wagner dans Tannhäuser, ainsi qu'au séjour de Luther (1521).

12. Le développement durable se situe à la confluence de trois préoccupations : économique. socialc ct cnvironncmentalc. Il vise à répondre aux besoins du présent sans compromettre les générations à venir.

13. Bundesamt für Bauwesen und Raumordnung; site officiel : http://www.bbr-bund.de 
kreise et une ville-Kreis. Certains sont même à cheval sur plusieurs régions d'aménagement comme le projet touristique «Forêt de Thuringe » ou le réseau de villes de Thuringe de l'Ouest. Enfin, des projets concernent plusieurs Länder : région Rhön (Thuringe, Bavière, Hesse), réseau de ville Sonneberg/Neustadt b. Coburg.

L'idée est de concevoir la concurrence régionale comme un vecteur de développement : les acteurs locaux proposent des projets innovants en valorisant des ressources locales spécifiques et en se distinguant des autres "régions ». Dans les faits, cette nouvelle démarche peut se révéler être un frein au développement et ceci, pour deux raisons. 
D'abord, comme on pouvait s'y attendre, des tensions naissent entre les projets régionaux et la région de planification. Prenons l'exemple de celles qui se manifestent entre l'Association de villes de Thuringe du Sud et la région de planification de Thuringe du Sud-Ouest. La première (Städteverbund Südthüringen) a été créée par six villes, rassemblées autour de Suhl (cf. Document 2), afin de prétendre au rang de ville centrale (ou Oberzentrum $)^{14}$.

Le projet du Städteverbund Südthüringen est fondé sur l'idée que, par la diversité du marché du travail, par le niveau et la qualité de vie, par les fonctions socio-économiques ainsi que par l'attractivité des centres urbains partenaires, ce réseau de villes pourrait constituer un pôle pour toute la région d'aménagement. Le fait d'être qualifié de «ville centrale » (Oberzentrum) par le Land permettrait d'envisager un développement économique plus dynamique et un rayonnement plus fort que ceux des villes moyenne (Mittelzentrum) ou petite (Unterzentrum). Par ailleurs, dans une logique de compétitivité, ce sont les villes centrales qui sont prioritaires pour l'obtention de subventions destinées à leur développement, que ce soit de la part du Land ou de l'État fédéral. Dans le cas de la Thuringe, seules trois villes sont actuellement reconnues comme «centrales »: Erfurt, Jena et Gera. La Thuringe du Sud-Ouest ne comportant que deux centres intermédiaires aux fonctions partielles de ville centrale (l'agglomération Suhl/Zella-Mehlis et Eisenach), c'est le projet non pas d'une «ville centrale » (Oberzentrum) mais d'un « réseau de villes faisant fonction de ville centrale » qui a été retenu. Il associe six localités - Suhl, Zella-Mehlis, Oberhof, Meiningen, Schmalkalden et Hildburghausen - qui, d'après une étude scientifique ${ }^{15}$, constituent un Oberzentrum théorique : l'addition de leurs potentialités en fait un centre de rang supérieur ou égal aux autres villes centrales de Thuringe (Erfurt, Jena, Gera).

Il faut toutefois que la région de planification et le Land reconnaissent et valident le projet pour que le réseau de villes puisse ensuite «se vendre ${ }^{16}$ comme le quatrième Oberzentrum de Thuringe. Or ce projet de réseau est perçu comme trop original par les aménageurs que nous avons pu rencon-

14. La vision de l'organisation de l'espace par les hommes politiques et les aménageurs est très marquée par le modèle christallérien, fondé sur la théorie des places centrales. Chaque $L$ and disposerait ainsi d'au moins une ville centrale de premier ordre (Oberzentrum); chaque région d'aménagement serait constituée autour d'un Oberzentrum ou d'une ville de rang secondaire (Mittelzentrum) disposant d'une partie des fonctions d'un Oberzentrum (exemples : Suhl et Eisenach); chaque Kreis serait organisé autour d'une ville de rang 3, etc.

15. Étude réalisée à la fin des années 1990 par le Pr. Dr. Hartmut Usbeck (Leipzig) et portant sur les éléments évoqués plus haut (qualité de la vie, marché du travail, etc.).

16. L'expression n'a été rencontrée qu'une fois au cours des entretiens mais elle reflète bien l'idée, évoquée plus haut, d'une interpénétration croissante du politique et de l'économie dans les formes de développement local et de gouvernances actuellement encouragées. 
trer aux échelons de la région de planification et du Land de Thuringe. En effet, il reviendrait à envisager non plus l'idée d'une « ville centrale » mais celle d'un « réseau de villes à fonction centrale ». Même si des structures semblables existent dans d'autres Länder, cette conception de l'Oberzentrum est inacceptable pour les représentants de la région de planification et du Land avec lesquels nous nous sommes entretenu : l'Oberzentrum n'est conçu que comme un point, une concentration de services et de population, et non comme un réseau au sein duquel les services seraient éparpillés. Un conflit entre une vision traditionnelle de l'aménagement et une vision plus novatrice peut donc être mis ici en évidence, la difficulté venant du fait que la deuxième ne peut s'épanouir que si elle obtient l'aval de la première. Si, officiellement, elle n'est toujours pas un « réseau de villes à fonction centrale ", l'Association de villes de Thuringe du Sud n'en continue pas moins d'exister. Elle correspond simplement, pour l'instant, à une forme plus courante de coopération intercommunale, centrée sur des initiatives, dont les plus récentes sont la création d'une zone d'activité de 400 ha, la constitution d'un réseau de transports en commun à l'échelle du Städteverbund, l'édition d'un catalogue commun de marketing régional destiné aux entreprises ou encore la diffusion d'une Regional-Card touristique donnant accès aux principaux sites et infrastructures de loisir. L'objectif final demeure néanmoins la constitution d'un Oberzentrum autour de ce réseau de villes.

Un autre élément conflictuel explique pourquoi cet objectif n'a pas été atteint jusqu'ici : la multiplication des projets, encouragée par l'État fédéral et le $B B R$, peut aboutir à des projets trop semblables pour servir de nouveau cadre au développement et susciter de vives tensions politiques. Il en est ainsi, par exemple, de l'Association de villes de Thuringe de l'Ouest, autour d'Eisenach, ville moyenne dynamique avec un riche patrimoine (Wartburg), davantage tournée dans la direction d'Erfurt que dans celle de la Thuringe méridionale ${ }^{17}$. Au moment où s'est formée l'Association de villes de Thuringe du Sud, le maire d'Eisenach a décidé d'élaborer son propre projet de réseau de villes. Lors de notre entretien, il l'a présenté comme « quasi factice » dans la mesure où il avait surtout pour but de contrecarrer le « réseau de villes à fonction centrale » voulu par la Thuringe du Sud. La perspective d'être dominé par un Oberzentrum autour de Suhl était inacceptable pour Eisenach... L'Association de villes de Thuringe de l'Ouest a, en conséquence, soumis elle aussi au Land le projet d'un Oberzentrum réticulaire. La stratégie a payé : le Land, déjà opposé à la conception d'un « réseau de villes à fonction centrale », a critiqué le fait que deux projets trop similaires soient envisagés dans une seule région de planification. Il n'en a donc accepté aucun.

17. Eisenach se situe en effet sur l'axe est-ouest reliant les principales villes de Thuringe (Eisenach, Erfurt, Weimar, Jena, Gera) ; voir le Document 1. 
DOCUMENT 2

Projets régionaux analysés dans la région de planification de Thuringe du Sud-Ouest

La multiplication des échelles et des projets régionaux entretient ainsi un certain flou et une relative incompatibilité des approches. Par ailleurs, la souplesse des cadres législatifs permet aux acteurs locaux d'user de stratégies, de jeux de concurrence conduisant, à la fois, à la formulation de projets novateurs et à l'utilisation - à des fins parfois très individualistes - des cadres traditionnels qui ont été maintenus. On ne peut toutefois pas en rester à l'idée d'un éclatement des structures de projets et à un échec de ce mode de développement régional. En effet, au-delà du caractère encore exploratoire de ces pratiques, nous avons pu observer, dans cette région de planification, une certaine cohérence des projets, fondée sur les relations entre les acteurs locaux. 


\section{Les ENJEUX De la RÉgionalisation des PROJETS : RÉSEAUX D'ACTEURS ET NOUVEAUX TERRITOIRES D'ACTION}

\subsection{UNE COHÉRENCE RÉGIONALE ASSURÉE PAR LES RÉSEAUX D'ACTEURS}

Au sein de la Thuringe du Sud-Ouest, les cinq principaux projets de développement analysés correspondent soit à des réseaux de villes autour d'espaces considérés comme fonctionnels et organisateurs de l'espace rural, soit à des régions de coopération autour d'entités paysagères qui se réfèrent à des espaces plus identitaires (cf. Document 2). Nous avons effectué vingt-quatre entretiens avec des acteurs importants du dévcloppcment régional : représentants de la région de planification et de l'aménagement à l'échelle du Land, principaux maires, Landräte, élus consulaires (chambre de commerce et d'industrie, chambre de l'artisanat), techniciens territoriaux (managers régionaux), etc. Comme l'illustre le Document 3, les personnes interrogées sont plus ou moins liées les unes aux autres dans le cadre d'un réseau régional d'acteurs, l'espace de référence qui les rassemble étant la région de planification (Thuringe du Sud-Ouest). Le schéma se fonde d'abord sur une typologie des relations unissant les différents acteurs interviewés en essayant d'éviter toute hiérarchisation sociale ou territoriale. Les liens identifiés sont de trois types : formalisés, réguliers ou informels, le tout aboutissant à un ensemble relativement complexe d'interrelations. Il est également possible de répartir les mêmes personnes en groupes d'acteurs en fonction de l'intensité de leurs relations. Si l'on se centre sur les relations formalisées et régulières, quatre grands cercles d'acteurs peuvent être identifiés. Surtout, le schéma met en évidence l'imbrication des différents projets autour d'un actcur rćgional central : lc Landrat à la tête du Landkreis de Schmalkalden-Meiningen, investi dans la plupart des projets régionaux comme dans la région de planification. Il est élu local (représentant d'un Kreis), président de la région de planification de Thuringe du Sud-Ouest, président du Regionalforum Thüringer Rhön (région Rhön), président du Regionalverbund Thüringer Wald (Forêt de Thuringe) et a été présenté par mes interlocuteurs comme le véritable initiateur du Städteverbund Südthüringen (Association de villes de Thuringe du Sud). Il est, en d'autres termes, l'acteur de la synthèse, le point de rencontre entre les différents projets de développement régional et le lien avec les anciennes pratiques d'aménagement au sein de la région de planification.

Si l'on élargit l'analyse à l'ensemble des acteurs du développement régional, on peut reprendre les propos de V. Lozac'h au sujet des expériences de démocratie locale, notamment à l'échelon de la commune : « La redéfinition des rapports entre État et société, consécutive à l'introduction de la démocratie, conduit à un redéploiement des initiatives privées qui tend à modifier les relations nouées à l'échelon local. Différents groupes d'acteurs sont désormais associés à la conduite des politiques communales. C'est en particulier le 
cas dans le domaine socio-culturel. Conformément au principe de subsidiarité en vigueur dans la République fédérale d'Allemagne, nombre de missions sont prises en charge par les structures associatives et caritatives. » (Lozac'h, 2001, pp. 168-169) Cette imbrication des initiatives privées et publiques est également de règle pour les projets de développement régional, l'expression «Public-Private-Partnerships» (PPP) étant l'un des leitmotive du Bundesamt für Bauwesen und Raumordnung. À l'échelon local, l'Association de villes de Thuringe du Sud est une illustration de ce mélange des genres dans le sens où quatre groupes de travail (Arbeitsausschüsse) thématiques (infrastructures, économie, culture/tourisme et protection contre les catastrophes) ont été créés en tant qu'instances de conseil auprès des élus, un peu sur le même modèle que les conseils de développement dans le cadre des Pays français ${ }^{18}$.

Il faut rappeler ici que, dans le contexte de l'Allemagne orientale, la constitution en cours des réseaux d'acteurs est à analyser à la lumière de l'héritage des pratiques centralisées et planifiées. Les responsables actuels du développement sont des acteurs majoritairement issus de la région étudiée et non pas des « professionnels » du développement transférés de l'Ouest vers l'Est. Le manager régional de l'Association de villes de Thuringe du Sud ainsi que le responsable de la région de planification de Thuringe du Sud-Ouest travaillaient tous deux pour les instances-relais de la planification territoriale à l'échelle du Bezirk de Suhl. Le premier a eu la possibilité (ou l'obligation ?) de suivre une formation en Bavière et en Hesse afin d'apprendre ce qu'était le développement local. Néanmoins, ce sont effectivement, à chaque fois, des acteurs locaux connaissant bien la région pour laquelle ils travaillent. À l'inverse, à l'échelon de l'aménagement du Land, son représentant à Weimar est un aménageur originaire de l'Ouest, transféré en Thuringe (avec un salaire réévalué pour l'occasion...). On retrouve ainsi, dans le cadre du développement local, un processus identique à celui qui a été observé lors de la réforme territoriale et administrative ayant suivi la réunification : «tandis qu'au niveau de l'État, un changement d'élites et un transfert d'élites d'Ouest en Est eurent lieu, le transfert fut nettement moindre au niveau communal » (Möller, 2001, p. 131). Il semble qu'il en soit allé de même au niveau « régional » ou local, même si les différences de structures et de pratiques entre les Länder doivent conduire à la plus grande prudence. La pluralité et la relative vivacité des projets de la Thuringe du Sud-Ouest ne se retrouvent vraisemblablement pas dans l'ensemble des régions de planification.

18. D'après la Loi d'orientation pour l'aménagement et le développement durable du territoire (1999), un Pays est un territoire de projets et un projet de territoire, mis en place par les acteurs locaux. Les élus constituent un bureau décisionnel. Parallèlement, il existe une instance de conseil qui regroupe des représentants économiques, associatifs ou encore consulaires : le conseil de développement. 
On peut donc en retenir que la Thuringe du Sud-Ouest est un cas intéressant du point de vue des projets de développement régional. Elle présente une cohérence fondée moins sur des territoires (de projets) que sur la mise en relation des acteurs locaux chargés de leur réalisation. En n'étant plus seulement un relais du Land mais une entité de coordination des projets et acteurs régionaux, la région de planification est un échelon cohérent en termes de réseaux d'acteurs, tout au moins en Thuringe du Sud-Ouest. On peut toutefois estimer que nombre d'autres régions de planification le sont également car les chercheurs et les aménageurs tendent à leur attribuer une fonctionnalité croissante (Sinz, 1995), notamment autour d'un modèle d'organisation de l'espace très christallérien ${ }^{19}$. La " planification de projet » (Selle, 1994), qui s'instaure actuellement en Allemagne et conçoit la région de planification comme une instance tant de planification que de liaison avec les projets locaux, mettrait dès lors en valeur un échelon qui serait

19. On a déjà mentionné, au début de cet article, la prégnance de la représentation christallérienne de l'espace parmi les politiques et les aménageurs en Allemagne. 
le reflet des institutions mais aussi, dans l'idéal, un territoire fonctionnel, voire identitaire. La région d'aménagement serait d'autant plus pertinente qu'elle coordonnerait des projets cohérents sur son territoire. À l'inverse, les projets de développement régional dont les limites spatiales ne sont pas nécessairement circonscrites seraient d'autant plus performants qu'ils s'inscriraient dans le cadre spatial délimité des régions de planification.

La cohérence d'acteurs assurée par la région de planification n'empêche pourtant ni l'élaboration de projets par delà les limites administratives ni la multiplication des projets régionaux ni, bien sûr, les concurrences et tensions évoquées précédemment. Les projets régionaux restent souvent très informels, volontairement ouverts pour garantir plus de souplesse. L'on peut y voir aussi un handicap car les capacités d'action sont d'autant plus limitées que les projets ne sont pas véritablement territorialisés. Par ailleurs, le poids de la région de planification pourrait, on l'a vu, freiner les projets les plus novateurs, notamment dans le cadre de réseaux de villes. Il est alors permis de penser que l'avenir des projets régionaux réside dans leur territorialisation, en liaison ou non avec la région d'aménagement.

\subsection{L'AVENIR DU DÉVELOPPEMENT RURAL EN AllemaGNE : \\ QUELS TERRITOIRES POUR LE DÉVELOPPEMENT?}

La conception du développement local en Allemagne se distingue en particulier de celle de la France où, dans le cas des espaces ruraux, la politique des Pays telle que l'ont définie les lois Pasqua (1995) et Voynet (1999) implique l'identification de territoires cohérents (culturels, économiques, sociaux ou géographiques) légitimant l'élaboration des projets (Giraud, 1999). Comme on l'a constaté, la question du développement régional en Allemagne repose plutôt sur la formation de réseaux d'acteurs détachés de tout cadre territorial strict, les approches étant souvent plus thématiques que territoriales (Quéva, 2007b). Elles ont l'avantage d'être suffisamment souples pour permettre la constitution de réseaux débordant les limites administratives. En revanche, si la démarche territoriale française a le mérite d'être plus transversale, elle tend aussi à promouvoir - avec des nuances dans un certain nombre de cas - un ancrage des projets de développement dans les territoires existants (le canton pour les communautés de communes et l'arrondissement pour les Pays). Cette territorialisation accrue semble parfois contraire à la logique initiale du projet par les acteurs locaux ${ }^{20}$ mais l'analyse de la réalité allemande montre qu'un trop grand détachement des structures territoriales fragilise la réalisation des projets de développement. Spécialement dans les nouveaux Länder, «l'action de l'État dans la transition des

20. De même, lors de nos entretiens menés en France, le poids grandissant des acteurs territoriaux traditionnels (maires, conseillers généraux) a très souvent été critiqué par les acteurs locaux issus de la société dite "civile ", de plus en plus souvent mis à l'écart des projets de développement. 
territoires ruraux de l'ex-Allemagne de l'Est vers l'économie de marché et l'autonomie locale va nécessairement de pair avec la réappropriation de ces territoires par les acteurs locaux » (Lacquement, 2001, p. 284).

Si la territorialité locale constitue un acquis à l'ouest de l'Allemagne, elle est plutôt de l'ordre de la (re-)découverte dans les espaces de l'ex-RDA. Ceux-ci doivent à la fois concilier les approches novatrices en termes de réseaux et la constitution de territoires locaux suffisamment forts pour ancrer ces réseaux. Plus généralement, l'articulation des réseaux d'acteurs et des territoires locaux est considérée comme une nécessité, aussi bien à l'échelle de l'État fédéral par les organismes d'aménagement qu'à l'échelle locale par les acteurs rencontrés dans la région de Thuringe du Sud-Ouest. À leurs yeux, les deux principales difficultés sont : (i) le trop grand nombre de projets régionaux, concurrents et insuffisamment coordonnés, qui implique le renforcement inévitable de la région d'aménagement comme outil de sélection et de coordination des projets ; (ii) la souplesse excessive de projets régionaux trop informels, qui requiert leur territorialisation par un ancrage dans des espaces de pouvoir et non plus seulement d'action. Pour le moment, la territorialisation n'est pas nécessairement affichée ou revendiquée. Dans la phase actuelle des échanges et des débats qui accompagnent les projets, ces derniers se caractérisent encore par leur ouverture et leur souplesse mais l'on peut supposer que la territorialisation s'imposera lors de leur réalisation.

En même temps, l'existence de spécificités régionales et l'appartenance à un ensemble géographique et culturel sous-tendent constamment l'élaboration des projets : la référence à l'entité de la Forêt de Thuringe, en particulier, est un élément fort du discours des acteurs autour de la notion de «patrimonialisation ». Selon certaines des personnes interrogées, la cohérence des actions devrait avoir pour cadre non la région d'aménagement mais celui, plus vaste, de la forêt de Thuringe, territoire identitaire et paysager associé à une culture et même à des dialectes spécifiques. Actuellement, un projet de développement régional est ainsi porté par les acteurs de huit Kreise répartis sur trois régions d'aménagement ${ }^{21}$ autour du thème du tourisme (mise en commun des ressources pour l'aménagement touristique, protection environnementale dans le cadre de parcs naturels). Parallèlement, l'un des principaux projets de la Chambre de commerce et d'industrie de Thuringe du Sud est de faire de cet espace, ainsi que du nom de « Forêt de Thuringe ", un label régional pour favoriser l'accueil d'entreprises. Il s'agirait d'élargir les compétences actuelles de la structure de coopération existante (regional Verbund Thüringer Wald) à d'autres domaines que le tourisme tels que la culture, la formation et le développement économique régional, ce qui supposerait la constitution d'une structure administrative plus aboutie, en liaison notamment avec les Landkreise. Une région iden- 
titaire et territorialisée, au moins symboliquement, peut donc être conçue comme le fondement d'un projet plus vaste de développement.

La concrétisation des projets, autrement dit le passage à l'acte, nécessite avant tout de recourir à des structures de pouvoir territorialisées pour obtenir des financements et mobiliser des acteurs capables de se faire entendre auprès des échelons supérieurs. Nous avons déjà évoqué le poids de l'échelon du Kreis à travers la figure du Landrat investi aussi bien dans la région de planification que dans la plupart des projets de développement régional analysés ici. De même, nos études de terrain à différentes échelles ont mis en évidence le rôle essentiel des territoires des Landkreise dans la réalisation des projets régionaux. À l'échelle de l'Association de villes de Thuringe du Sud (Städteverbund Südthüringen), les acteurs rencontrés ont certes toujours insisté sur les relations entre les six villes et l'idée d'une région centrale réticulaire, quasiment détachée de toute structure territoriale. Dans les faits cependant, l'espace concerné par les initiatives du Städteverbund ne se borne pas au territoire communal des six villes mais comprend toutes les communes situées dans la ville-Kreis de Suhl et dans les deux Landkreise. Le champ d'action du réseau correspond ainsi à l'ensemble des territoires de ses Kreise d'appartenance. De même, dans la région $\mathrm{Rhön} \mathrm{(réserve} \mathrm{de} \mathrm{biosphère),} \mathrm{un} \mathrm{réseau} \mathrm{d'acteurs} \mathrm{s'est} \mathrm{mis} \mathrm{en} \mathrm{place}$ par delà les limites des Länder mais, concrètement, les six Kreise dont le territoire renferme une partie de l'entité paysagère de la Rhön s'en partagent la gestion. À l'échelle de la région de planification, il est logique que les élus des Kreise jouent un rôle central étant donné que celle-ci regroupe plusieurs Kreise. Enfin, à l'échelle régionale la plus vaste - celle, mentionnée supra, des projets de développement reposant sur l'entité paysagère de la Forêt de Thuringe -, les principaux responsables de la gestion de cet espace sont également les huit Landkreise et les deux villes-Kreis dont le territoire comprend une partie de la forêt de Thuringe.

Ces trois exemples sont emblématiques d'un certain « retour » au territoire dans les expériences de développement régional. La question d'une réforme territoriale des communes ou des Kreise, qui pourrait d'ailleurs succéder assez rapidement à cette phase exploratoire de projets, a été très souvent évoquée par les acteurs locaux durant nos entretiens. Ils font preuve, à cet égard, d'une relative ouverture d'esprit, ayant vécu de manière abrupte et rapide différentes réformes territoriales et administratives depuis la réunification.

$$
* * *
$$

L'ensemble de ces expériences montre le caractère encore exploratoire des démarches endogènes de développement. On est aujourd'hui, en Allemagne, à la recherche d'une échelle pertinente pour l'action et d'une articulation des espaces fonctionnels, identitaires et institutionnels. La difficulté réside dans la capacité des acteurs à conjuguer harmonieusement 
les anciennes et les nouvelles échelles de l'aménagement car il n'est pas question, pour l'instant, d'en supprimer. Globalement, un souci d'élargissement des échelles classiques se manifeste : de la commune à la coopération intercommunale, du Kreis à la coopération régionale et de la région d'aménagement à la coopération interrégionale. Ces évolutions sont assez semblables à celles que connaît la France avec le passage de l'échelle communale aux communautés de communes, du canton au Pays, voire du département à la région. À l'arrière-plan, c'est peut-être un modèle européen qui se met en place, valorisant de nouveaux territoires aux échelles régionale et locale. Ceci n'est qu'une hypothèse que des recherches comparatives plus approfondies et plus systématiques pourraient valider ${ }^{22}$.

\section{RÉFÉRENCES BIBLIOGRAPHIQUES}

Alvergne C. \& Tautelle F. (2002), Du local à l'Europe. Les nouvelles politiques d'aménagement du territoire, Paris : PUF, 304 p.

Baudelle G. \& KunzmanN K.-R. (2004), « Regards croisés sur l'aménagement du territoire en France et en Allemagne », ESO, travaux et documents, $\mathrm{n}^{\circ} 22$, pp. 69-77.

BönisCh R., Mohs G. \& Ostwald W. (1980), Territorialplanung, Berlin: Verlag Die Wirtschaft, 288 p.

Bundesamt für Bauwesen und Raumordnung (1999), « Perspektiven für die Region als Planungs- und Handlungseben », Informationen zur Raumentwicklung, Heft 9/10, pp. 577-712.

Diller C. (2002), «Zwischen Netzwerk und Organisation. Die Dynamik der Versteigerung regionaler Kooperationen ", Raumforschung und Raumordnung, Heft 2, pp. 146-154.

Felgenhauer T., Minm M. \& Schlottmann A. (2003), « Langage, médias, et régionalisation symbolique. La fabrication de la Mitteldeutschland », Géographie et culture, $\mathrm{n}^{\circ} 47$, pp. 85-102.

FürsT D. (2001), « Regional governance - ein neues Paradigma der Regionalwissenschaften? ", Raumforschung und Raumordnung, Heft 5-6, pp. 370-380.

Fürst D. \& SCHUBert H. (2001), « Regionale Akteursnetzwerke zwischen Bindungen und Optionen. Über die informelle Infrastruktur des Handlungssystems bei der Selbstorganisation von Regionen », Geographische Zeitschrift, Heft 1,pp. 32-51.

22. Cette hypothèse fait l'objet de mes recherches actuelles. Voir G. Vergnaud et C. Queva, L'" intermédiarité » des territoires locaux : essai de réflexions croisées sur les constructions territoriales locales en Allemagne, en France et en Espagne; communication au colloquc «Territoires, territorialité, territorialisation : et après ? (Grenoble, Cité des territoires, PACTE-territoires, UMR 5194, 7-8 juin 2007). 
GiRAud F. (1999), «LOADDT : le sacre de l'approche territoriale et de la relégation de l'approche spatiale », Montagnes méditerranéennes, ${ }^{\circ} 9$, Université de Grenoble, pp. 41-43.

GrefFe X. (2002), Le développement local, La Tour d'Aigues : DATAR-Éditions de l'Aube, 199 p.

GUDER U. (2003), L'aménagement du territoire et la politique régionale en Allemagne : vers une européanisation en douceur?, Groupement d'études et de recherches « Notre Europe », 47 p.

LACOUEMENT G. (2001), «L'État et la décollectivisation dans les nouveaux Länder allemands », in A.-M. Le Gloannec, dir., L'État en Allemagne. La République fédérale après la réunification, Paris : Presses de Sciences Po, pp. 265-284.

Lacouement G. (2004), «Politique d'intégration territoriale et ancienne frontière interallemande ", Annales de géographie, ${ }^{\circ}$ 636, pp. 168-187.

LoZAC'H V. (2001), « La démocratie locale dans les nouveaux Länder », in A.M. Le Gloannec, dir., L'État en Allemagne. La République fédérale après la réunification, Paris : Presses de Sciences Po, pp. 152-182.

Möller B. (2001), « Administration municipale et acteurs communaux dans le processus de transformation d'Allemagne de l'Est », in A.-M. Le Gloannec, dir., L'État en Allemagne. La République fédérale après la réunification, Paris : Presses de Sciences Po, pp. 129-149.

QuÉVA C. (2007a), « Les paradoxes de la Region en Allemagne, entre réseaux et territoires : la région, outil de déterritorialisation ? ", Annales de géographie, ${ }^{\circ}$ 653, pp. 41-64.

QuÉva C. (2007b), « La ressource territoriale en question dans les logiques de requalification contemporaine des territoires locaux en Allemagne », in H. Gumuchian \& B. Pecqueur, dir., La ressource territoriale, Paris : Economica/Anthropos, pp. 126-132.

SELLE K. (1994), Was ist bloss mit der Planung los? Erkundungen auf dem Weg zum kooperativen Handels. Ein Werkbuch, Dortmund: IRPUD, 365 p.

SiNZ M. (1995), "Region », in Akademie für Raumforschung und Landesplanung, Handwörterbuch der Raumordnung, Braunschweig: VSB, pp. 805-808.

VANIER M. (2002), «Les espaces du politique : trois réflexions pour sortir des limites du territoire », in B. Debarbieux \& M. Vanier, dir., Ces territorialités qui se dessinent, La Tour d'Aigues : DATAR-Éditions de l'Aube, pp. 75-89.

Wollmann H. (1996), « The Transformation of Local Government in East Germany: Between Imposed and Innovative Institutionalization », in A. Benz \& K. Goetz, eds., A New German Public Sector, Altershot: Dartmouth Publishing Co, pp. 137-163. 